\title{
Heterogeneous prognoses of patients with tumors invaded within muscularis propria according to tumor depth in the layers of the muscularis propria
}

\author{
Yu Imamura ${ }^{1}$, Yoshifumi Baba ${ }^{1,2}$, Shinji Ishikama ${ }^{1}$, Yukiharu Hiyoshi ${ }^{1}$, Youhei Nagai ${ }^{1}$, Tadahiko Nakamura $^{1}$, \\ NaOko Hayashi ${ }^{1}$, NobUtomo Miyanari ${ }^{1}$, Kenichi Iyama $^{2}$, and Hideo BabA ${ }^{1}$ \\ ${ }^{1}$ Department of Gastroenterological Surgery, Graduate School of Medical Sciences, Kumamoto University, 1-1-1 Honjo, Kumamoto 860-8556, \\ Japan \\ ${ }^{2}$ Department of Surgical Pathology, Kumamoto University Hospital, Kumamoto, Japan
}

\begin{abstract}
Background. The muscularis propria of the stomach is histologically divided into three layers; namely, the innermost oblique, the inner circular, and the outer longitudinal layers. In patients with gastric cancer the depth of tumor invasion has been reported to correlate with lymph node metastasis and prognosis . However, it is unclear whether the depth of tumor invasion in the muscularis propria has an effect on lymph node metastasis and prognosis.

Methods. Fifty-nine gastric cancer patients with muscularis propria invasion were analyzed retrospectively. These patients were divided into two groups, the inner group, with invasion up to the inner circular layer; and the outer group, with invasion beyond the inner circular layer. The relationships between tumor invasion and clinicopathological factors and survival were evaluated.

Results. Of the 59 patients, 34 were classified as the inner group, and 25 were classified as the outer group. The inner group had a significantly lower probability of lymph node metastasis $(P=0.0053)$ and a significantly better overall cancer-specific survival $(P=0.017)$ than the outer group.

Conclusion. Gastric cancers with muscularis propria invasion had heterogeneous prognoses according to the tumor depth in the muscularis propria layers.
\end{abstract}

Key words Gastric cancer- Muscularis propria· Prognosis

\section{Introduction}

Gastric cancer is a common neoplasm worldwide. Though an $\mathrm{R} 0$ resection can be performed in most cases, advanced gastric cancer (AGC) still often recurs. In Japan, AGC is defined as a tumor with invasion beyond the muscularis propria (MP), regardless of the presence

Offprint requests to: $\mathrm{H}$. Baba

Received: November 7, 2007 / Accepted: October 7, 2008 or absence of lymph node metastases. Patients with gastric cancer with invasion within the MP (MP gastric cancer) in Japan account for about $10 \%$ of all gastric cancer patients undergoing a surgical resection [1, 2]. MP gastric cancer is usually treated by a curative surgical resection, and the 5-year survival rate was reported as $72 \%$ to $82 \%$ [3, 4]. Although most patients with MP gastric cancer are cured by an $\mathrm{R} 0$ resection, $16 \%$ to $22 \%$ of these patients have recurrences postoperatively $[3,5]$.

There are many reports that the subclassification of gastric cancer with invasion within the submucosal layer (SM gastric cancer) is useful to predict lymph node metastasis [6, 7]. It remains unclear, however, whether subdivision of tumor invasion into the MP is useful for predicting lymph node metastasis and prognosis $[8,9]$. In general, the MP is histologically divided into three layers; namely, the innermost oblique, the inner circular, and the outer longitudinal layers. Actually it is difficult to distinguish the innermost oblique and inner circular layers of the stomach under a microscope. However, it is easier to distinguish the inner circular and outer longitudinal layers. These results and facts suggest that the subdivision of MP gastric cancer into cancers in the inner circular and outer longitudinal layers might therefore make it possible to predict the prognosis of patients with MP gastric cancer. The present study evaluated the influence of the extent of tumor invasion into the MP.

\section{Patients and methods}

From 1983 to 2002, 798 consecutive patients underwent gastrectomy with lymph node resection for primary gastric cancer at Kumamoto University Hospital. Of these 798 patients, $72(9.0 \%)$ patients had invasion within the MP. In 60 of these 72 patients, clinical data, including the surgical records, the histological findings, 


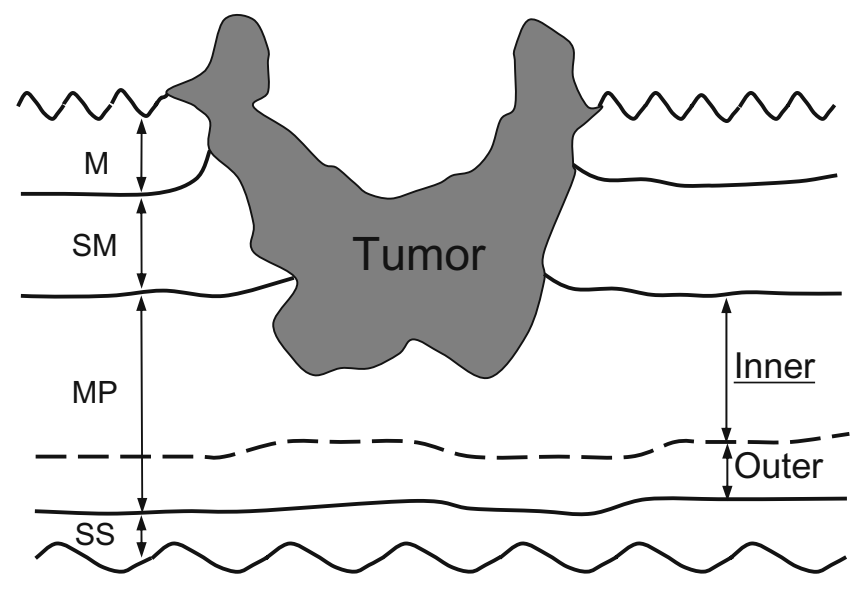

Fig. 1. The muscularis propria $(M P)$ is divided by the dotted line. $M$, Mucosa; $S M$, submucosa; $S S$, subserosa

the long-term survival, and disease free survival were available. One patient with a lymph node metastasis regarded as a distant metastasis according to the Japanese Gastric Cancer Association Japanese classification of gastric carcinoma (Japanese classification) [10] was excluded from this study. The remaining $59 \mathrm{MP}$ gastric cancer patients were eligible, and their median followup period was 77 months (range, 6-189 months).

For histological examinations, $10 \%$ formalin-fixed, paraffin-embedded specimens were cut into 3-mm-thick sections. In each case, cancer invasion was evaluated using the section with the deepest invasion stained with hematoxylin-eosin (H\&E). For the evaluation of venous invasion and lymphatic invasion, Victoria blueH\&E staining and immunohistochemical staining for D2-40 (Nichirei, Tokyo, Japan) [11], respectively, were carried out.

The 59 patients were divided into two groups according to the depth of tumor invasion. The patients with invasion up to the inner circular layer were classified as the inner group, and the patients with invasion beyond the inner circular layer were classified as the outer group. In addition, patients in whom the cancer invasion front was located between the inner circular and outer longitudinal layers were classified as the outer group. A schematic drawing of the MP and the subdivision into inner and outer layers is shown in Fig. 1, and the schema and examples from each group are shown in Fig. 2. The subdivision was carried out by two pathologists without any knowledge of either the clinical or survival data.

The $\chi^{2}$ test was used to compare data between the inner and outer groups, and the Kaplan-Meier method and log-rank test were used to analyze the patients' postoperative survival. A $P$ value of less than 0.05 was considered to be statistically significant.

\section{Results}

Of the 59 gastric cancer patients with MP invasion, 34 were classified as the inner group, and 25 were classified as the outer group. The comparison of the clinicopathological factors in MP gastric cancer patients by the subdivision is summarized in Table 1. The outer-group patients had significantly higher probabilities of lymph node metastasis and venous invasion than the inner group ( $P=0.0053$ and $P=0.027$, respectively).

The 5-year cancer-specific survival rates of the inner and outer groups were $94 \%$ and $72 \%$, respectively. Univariate analyses revealed that the only statistically significant prognostic factor in patients with MP gastric cancer was the subdivision of the MP $(P=0.017$; Table $2)$. The inner-group patients had a significantly better prognosis than the outer group (Fig. 3). Patients with lymph node metastasis tended to have poor prognoses; however, this factor did not reach statistical significance in this study $(P=0.067$; Table 2$)$.

The recurrence rate of the inner group was $6 \%(2 / 34)$ and that of the outer group was 36\% (9/25). The difference between these two groups was statistically significant $(P=0.0082$; Table 3$)$. Regarding the recurrent sites, two inner-group patients had peritoneal recurrences. In the eight outer-group patients with recurrences, four patients had liver recurrences, two patients had peritoneal recurrences, one patient had lymph node recurrence, and one patient had bone recurrence. This result suggests that the difference in the recurrence rates might thus have resulted in the difference between the clinical outcomes in the two groups.

\section{Discussion}

Even if histologically diagnosed as having MP gastric cancer, which is an advanced disease, some patients nevertheless still have a good clinical outcome. Histologically, the MP is divided into three layers, the innermost oblique, the inner circular, and the outer longitudinal layers. The gastric innermost oblique layer appears from the esophageal inner layer and does not reach the antrum. The gastric inner circular layer is the thickest layer, and is well-muscled, especially in the pylorus as the sphincter pylori muscle. The gastric outer longitudinal layer leads from the esophageal outer muscular layer, and leads to the duodenal outer longitudinal layer. In this study, we hypothesized that the subdivision of MP gastric cancer according to these layers might help to predict the prognosis of patients with MP gastric cancer, as reported in the subclassification of SM gastric cancer. In subdividing the MP, the lowest line of the inner circular layer was selected as the boundary line, because it is easier to distinguish the inner circular and 

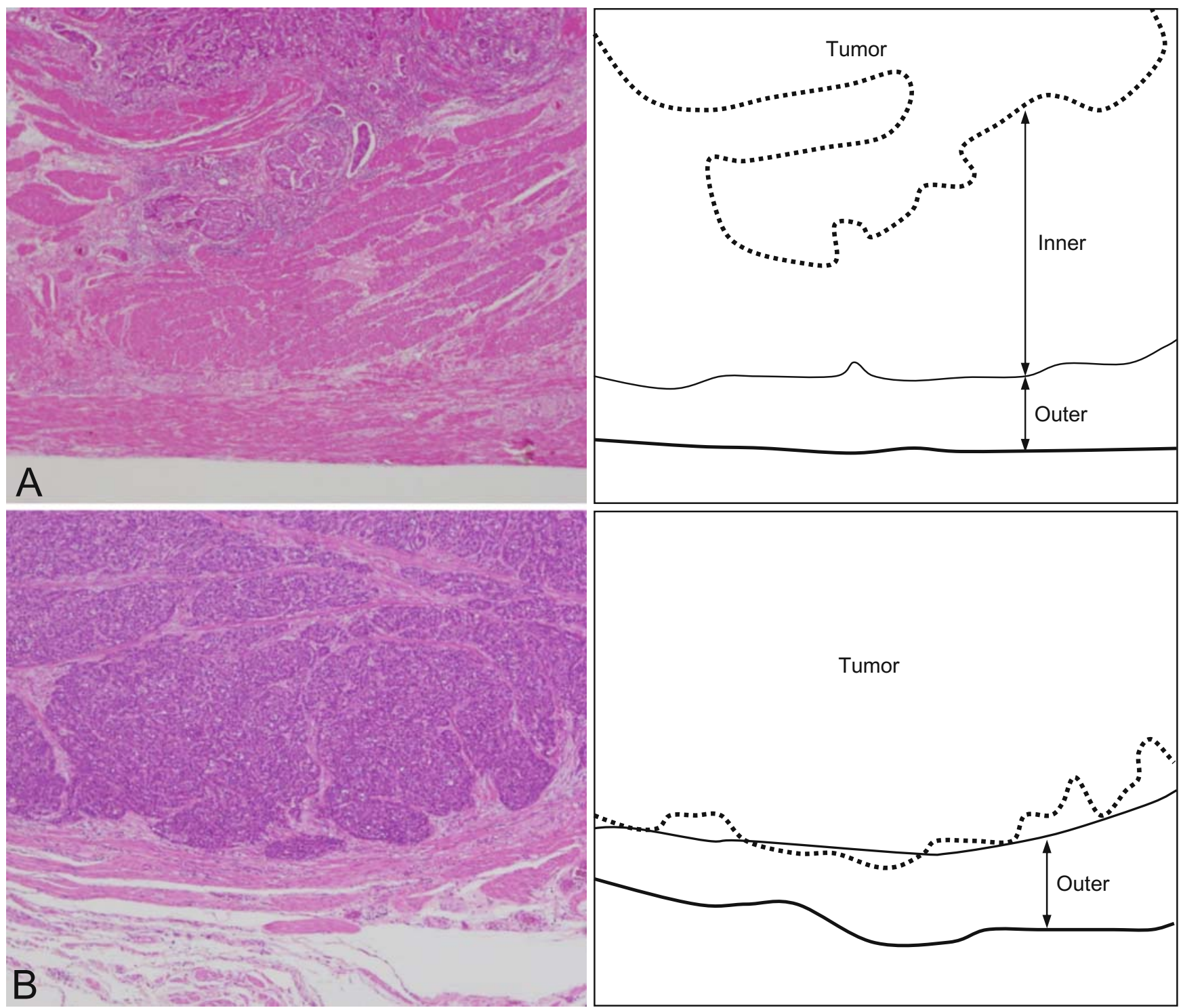

Fig. 2A, B. In the schemas on the right, the dotted lines show the cancer invasion front. The solid lines show the area of the outer longitudinal layer; the upper solid lines show the lowest line of the inner circular layer (the boundary line). A Micrograph on the left shows a case that was classified into the inner

outer longitudinal layers than it is to distinguish the innermost oblique and inner circular layers under a microscope. In the present study, the results indicate that the subdivision of MP gastric cancer can help to predict patients' prognoses.

Ishigami et al. [8] subclassified MP gastric cancer into two types according to the depth of tumor invasion. They divided the MP layer into two types as follows: MP1, in which the tumor was limited to the upper third layer, and MP2, in which the tumor had expanded beyond MP1. MP1 cases had a significantly lower frequency of lymph node metastasis and a higher rate of

group. H\&E, $\times 40$. The tumor cells exist within the inner circular layer. B Micrograph on the left shows a case that was classified into the outer group. The tumor cells are observed to invade the outer longitudinal layer. $H \& E, \times 40$

operative cure than MP2 cases. However, the procedure used by Ishigami et al. [8] for subdividing MP gastric cancers in order to measure the depth of invasion was quite complex. We note the following issues: first, the volume of the invaded tumor and the associated inflammation make the layers (mucosa, submucosa [SM], and MP) of the cancer lesion thicker than the normal layers, and it is difficult for pathologists to measure the MP layer. Second, in cases where the border is between MP1 and MP2, the pathologist must measure its accurate depth, and this requires extensive time and effort. Histologically, there are three layers in the MP of the 
Table 1. Comparison of clinicopathological factors in gastric cancer patients with muscularis propria invasion according to subdivision of muscularis propria layers

\begin{tabular}{|c|c|c|c|c|}
\hline \multirow[b]{2}{*}{ Factors } & \multicolumn{2}{|c|}{ Invasion } & \multirow[b]{2}{*}{ Total $(n=59)$} & \multirow[b]{2}{*}{$P$ value } \\
\hline & Inner $(n=34)$ & Outer $(n=25)$ & & \\
\hline Mean age (years) & $64 \pm 13$ & $63 \pm 12$ & & 0.65 \\
\hline Sex & & & & 0.83 \\
\hline Male & 24 & 17 & 41 & \\
\hline Female & 10 & 8 & 18 & \\
\hline Tumor location & & & & 0.14 \\
\hline Upper & 4 & 8 & 12 & \\
\hline Middle & 17 & 11 & 28 & \\
\hline Lower & 13 & 6 & 19 & \\
\hline Tumor size (mm) & $44 \pm 25$ & $41 \pm 20$ & & 0.51 \\
\hline Histological grading & & & & 0.86 \\
\hline Differentiated & 21 & 16 & 37 & \\
\hline Undifferentiated & 13 & 9 & 22 & \\
\hline Nodal involvement & & & & 0.0053 \\
\hline Negative & 22 & 7 & 29 & \\
\hline Positive & 12 & 18 & 30 & \\
\hline Lymphatic invasion & & & & 0.50 \\
\hline Negative & 12 & 11 & 23 & \\
\hline Positive & 22 & 14 & 36 & \\
\hline Venous invasion & & & & 0.027 \\
\hline Negative & 28 & 11 & 39 & \\
\hline Positive & 6 & 14 & 20 & \\
\hline Adjuvant chemotherapy & & & & 0.13 \\
\hline No & 11 & 13 & 24 & \\
\hline Yes & 23 & 12 & 35 & \\
\hline
\end{tabular}

Table 2. Univariate analyses of prognostic markers in patients with gastric cancer with muscularis propria invasion

\begin{tabular}{|c|c|c|c|}
\hline Variable & Number of patients & 5-Year survival (\%) & $P$ value \\
\hline Age (years) ${ }^{a}$ & & & 0.33 \\
\hline$<66$ & 28 & 89.3 & \\
\hline$\geq 66$ & 31 & 80.6 & \\
\hline Sex & & & 0.68 \\
\hline Male & 41 & 85.4 & \\
\hline Female & 18 & 83.3 & \\
\hline Tumor location & & & $-^{\mathrm{b}}$ \\
\hline Upper & 12 & 73.7 & \\
\hline Middle & 28 & 100 & \\
\hline Lower & 19 & 66.7 & \\
\hline Tumor size $(\mathrm{mm})^{\mathrm{a}}$ & & & 0.91 \\
\hline$<35$ & 27 & 85.2 & \\
\hline$\geq 35$ & 32 & 84.4 & \\
\hline Histological grading & & & 0.85 \\
\hline Differentiated & 37 & 83.8 & \\
\hline Undifferentiated & 22 & 86.4 & \\
\hline Nodal involvement & & & 0.067 \\
\hline Negative & 29 & 93.1 & \\
\hline Positive & 30 & 76.7 & \\
\hline Lymphatic invasion & & & 0.80 \\
\hline Negative & 23 & 82.6 & \\
\hline Positive & 31 & 86.1 & \\
\hline Venous invasion & & & 0.32 \\
\hline Negative & 42 & 88.1 & \\
\hline Positive & 17 & 76.1 & \\
\hline MP subdivision & & & 0.017 \\
\hline Inner MP & 34 & 94.1 & \\
\hline Outer MP & 25 & 72.0 & \\
\hline Adjuvant chemotherapy & & & 0.71 \\
\hline No & 24 & 83.3 & \\
\hline Yes & 35 & 85.7 & \\
\hline
\end{tabular}

MP, muscularis propria

${ }^{a}$ Cutoff value was defined as the median value

${ }^{\mathrm{b}} P$ value can not be calculated because middle group patients did not die 
Table 3. Recurrences after surgery in muscularis propria gastric cancer

\begin{tabular}{|c|c|c|c|c|}
\hline \multirow[b]{2}{*}{ Mode of recurrence } & \multicolumn{2}{|c|}{ Invasion } & \multirow[b]{2}{*}{ Total $(n=59)$} & \multirow[b]{2}{*}{$P$ value } \\
\hline & Inner $(n=34)$ & Outer $(n=25)$ & & \\
\hline Recurrence & & & & 0.0082 \\
\hline Negative & 32 & 17 & 49 & \\
\hline Positive & 2 & 8 & 10 & \\
\hline \multicolumn{5}{|l|}{ Recurrent site } \\
\hline Liver & 0 & 4 & 4 & \\
\hline Bone & 0 & 1 & 1 & \\
\hline Peritoneum & 2 & 2 & 4 & \\
\hline Lymph node & 0 & 1 & 1 & \\
\hline
\end{tabular}

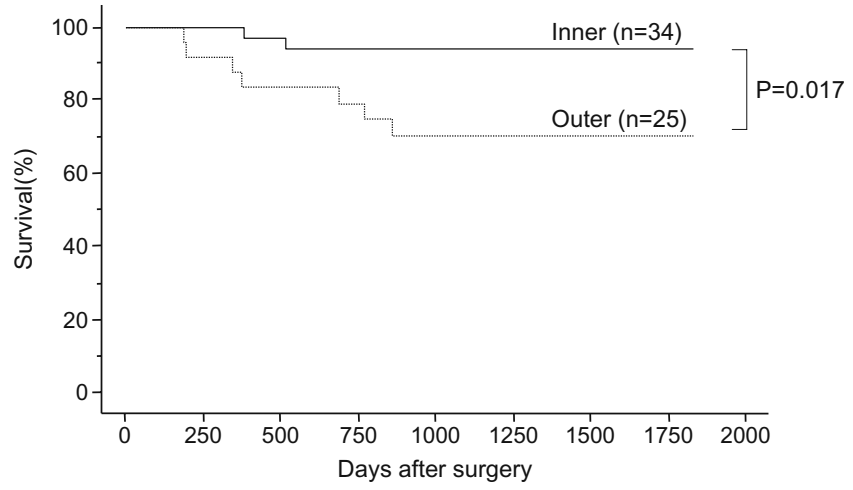

Fig. 3. Survival curves of patients with MP gastric cancer. The inner group had a significantly better prognosis than the outer group $(P=0.017)$

stomach, as mentioned above. Pathologists can easily detect the lowest line of the inner circular layer and subdivide MP gastric cancer using this dividing line without measuring the depth. This subdivision of the MP layer correlates with lymph node metastasis and prognosis, and this method is useful to predict the risk of recurrence.

In the present study, the inner group had a significantly better prognosis than the outer group. The mean thickness of the inner circular layer (obtained by adding the thicknesses of the innermost oblique and inner circular layers) was about three and a half times the thickness of the outer longitudinal layer in the normal part of the stomach (MP, $1822 \pm 676 \mu \mathrm{m}$; the innermost oblique + inner circular layers, $1353 \pm 560 \mu \mathrm{m}$; the outer longitudinal layer, $382 \pm 230 \mu \mathrm{m}$ ). Though it is natural that when the tumor invasion is deeper, the tumor has a greater chance of vessel invasion, a plexus of lymphatic vessels was noted between the inner circular and outer longitudinal layers (Fig. 4). In this myenteric region, there is a plexus of nerves, lymphatic ducts, and blood vessels. In recent studies, the interstitial cells of Cajal (ICC) in this myenteric region have been widely observed, and they are reported to form a network that is associated with the complicated movements of the smooth muscle of the stomach $[12,13]$. When the tumor invades this plexus, the rate of lymph node metastasis becomes higher and this causes a high frequency of lymph node metastasis. The presence of cancer cells in the lymphatic ducts and blood vessels in this myenteric region is definitely associated with the prognosis. Son et al. [14] divided MP gastric cancer into inner and outer levels and reported that the depth of invasion within MP gastric cancer was associated with no difference between these two groups in the patients' prognoses. But they did not show which line of the layers they used to divide the three layers into two groups and which level included the myenteric plexus, nor did they mention the existence of the lymphatic plexus. Indeed, in the present study, there were nine patients with tumor invasion reaching the plexus.

At present in Japan, patients with pathological T2 gastric cancer with lymph-node metastasis are recommended to receive adjuvant chemotherapy [15]. It may be suggested that inner-group patients with lymph-node metastasis need not receive adjuvant chemotherapy, because the 5-year survival of this group in the present study was $91.7 \%$ and it was significantly better than that of the other T2 patients (excluding the inner-group patients) with lymph-node metastasis (data not shown). However, this remains no more than speculation, as this study was retrospectively designed, and the sample size was too small to perform a multivariate analysis.

In conclusion, whether the tumor reaches this plexus of lymphatic vessels between the inner circular and outer longitudinal layers might be one of the most important factors for predicting the prognosis of patients with MP gastric cancer. If the tumor invasion is only up to the inner circular layer of the MP of the stomach, then the patients have a good chance to achieve a good clinical outcome.

Acknowledgments We thank H. Taniguchi for cooperation with the staining. 


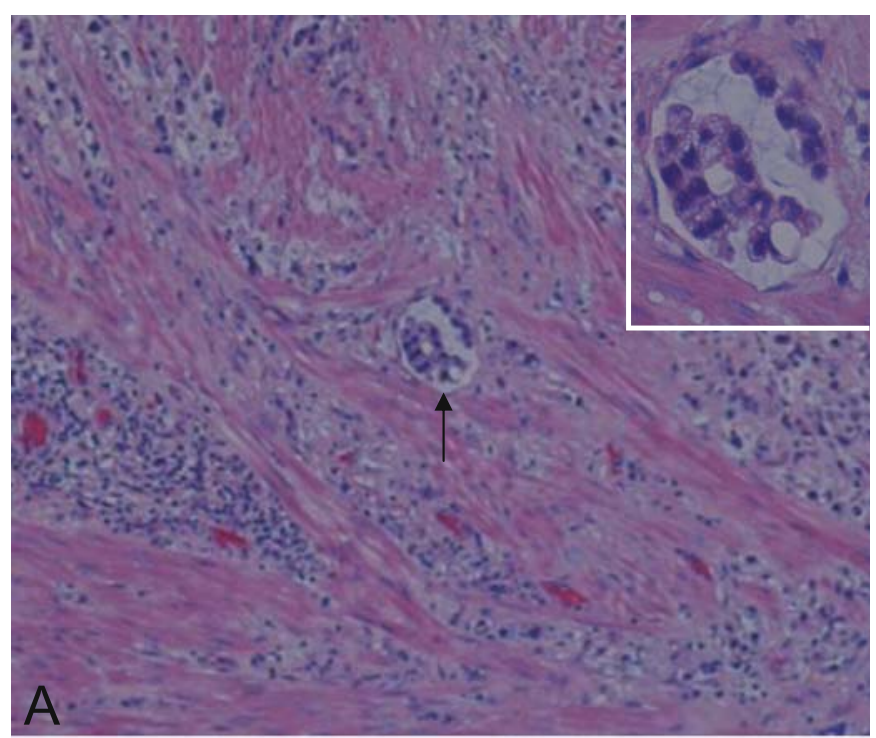

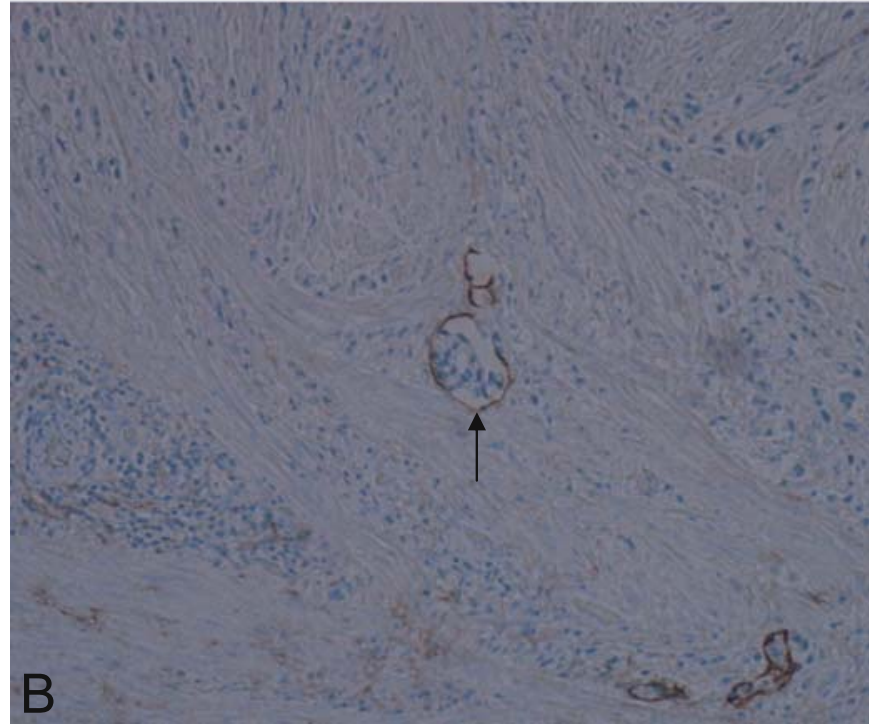

Fig. 4A, B. The plexus of invaded lymphatic vessels between the inner circular and outer longitudinal muscular layers. In the schema in $\mathbf{B}$, the solid line shows the lowest line of the inner circular layer (the boundary line). The dotted line shows the upper edge of the outer longitudinal layer. A Micrograph

\section{References}

1. Yoshikawa K, Maruyama K. Characteristics of gastric cancer invading to the proper muscle layer with special reference to mortality and cause of death. Jpn J Clin Oncol 1985;15:499-503.

2. Noguchi Y, Imada T, Matsumoto A, Coit DG, Brennan MF. Radical surgery for gastric cancer. A review of the Japanese experience. Cancer 1989;64:2053-62.

3. Yokota T, Kunii Y, Teshima S, Yamada Y, Saito T, Kikuchi S, et al. Gastric cancer with invasion limited to the muscularis propria. Int Surg 1999;84:7-12.

4. Otsuji E, Kuriu Y, Ichikawa D, Okamoto K, Hagiwara A, Yamagishi $\mathrm{H}$. Characteristics of gastric carcinoma invading the muscularis propria. J Surg Oncol 2005;92:104-8.

5. Takeuchi H, Kakeji Y, Tokunaga E, Koga T, Maehara Y, Sugimachi K. Prediction of recurrence after curative resection of

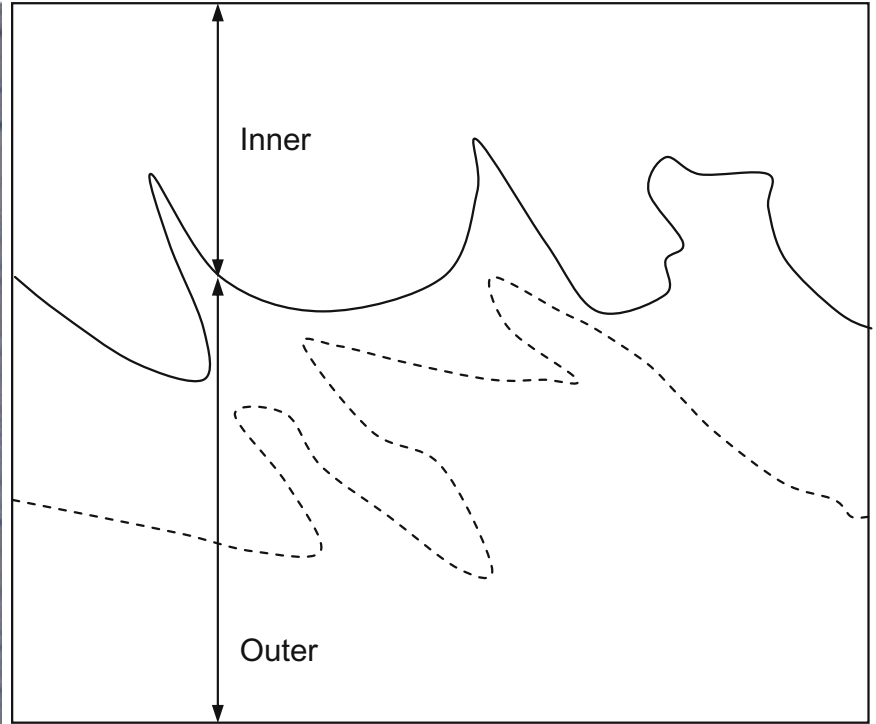

shows stomach wall with H\&E staining, $\times 100$. B Micrograph shows the same section as that in $\mathbf{A}$ immunohistochemically stained using monoclonal antibody D2-40. $\times 100$. Arrows in $\mathbf{A}$ and $\mathbf{B}$ show the invaded lymphatic plexus

gastric carcinoma invading the muscularis propria: a multivariate analysis. Gastric Cancer 2000 4;3:28-32.

6. Ichikura T, Uefuji K, Tomimatsu S, Okusa Y, Yahara T, Tamakuma S. Surgical strategy for patients with gastric carcinoma with submucosal invasion. A multivariate analysis. Cancer 1995;76:935-40.

7. Abe N, Sugiyama M, Masaki T, Ueki H, Yanagida O, Mori T, et al. Predictive factors for lymph node metastasis of differentiated submucosally invasive gastric cancer. Gastrointest Endosc 2004;60:242-5.

8. Ishigami S, Natsugoe S, Miyazono F, Hata $\mathrm{Y}$, Uenosono $\mathrm{Y}$, Aikou T, et al. Clinical merit of subdividing gastric cancer according to invasion of the muscularis propria. Hepatogastroenterology 2004;51:869-71.

9. Maehara Y, Anai H, Moriguchi S, Watanabe A, Tsujitani S, Sugimachi K. Gastric carcinoma invading muscularis propria and macroscopic appearance. Eur J Surg Oncol 1992;18:131-4. 
10. Japanese Gastric Cancer Association. Japanese classification of gastric carcinoma — 2nd English edition. Gastric Cancer. 1998;1: $10-24$.

11. Yonemura Y, Endou Y, Tabachi K, Kawamura T, Yun HY, Kameya T. Evaluation of lymphatic invasion in primary gastric cancer by a new monoclonal antibody D2-40. Hum Pathol 2006; 37:1193-9.

12. Cousins HM, Edwards FR, Hickey H, Hill CE, Hirst GD. Electrical coupling between the myenteric interstitial cells of Cajal and adjacent muscle layers in the guinea-pig gastric antrum. J Physiol 2003;550:829-44.
13. Ordog T, Ward SM, Sanders KM. Interstitial cells of Cajal generate electrical slow waves in the murine stomach. J Physiol 1999; 518:257-69.

14. Son HJ, Myung W, Yoo HS, Park SH, Song SY, Kwon YD, et al. Prognostic indicators of gastric carcinoma confined to the muscularis propria. Histopathology 2007;51:105-10.

15. Japanese Gastric Cancer Association. Gastric cancer treatment guidelines for doctors' reference (in Japanese). Tokyo: Kanehara; 2004. 